\title{
Mucinous Cystadenoma of the Testis: A Case Report with Immunohistochemical Findings
}

\author{
Gilhyang $\mathrm{Kim}^{1}$ - Dohee Kwon ${ }^{1}$ \\ Hee Young $\mathrm{Na}^{1}$. Sehui $\mathrm{Kim}^{1}$ \\ Kyung Chul Moon ${ }^{1,2}$ \\ ${ }^{1}$ Department of Pathology, ${ }^{2}$ Kidney Research \\ Institute, Medical Research Center, Seoul \\ National University College of Medicine, Seoul, \\ Korea \\ Received: June 29, 2016 \\ Revised: August 18, 2016 \\ Accepted: August 30, 2016 \\ Corresponding Author \\ Kyung Chul Moon, MD \\ Department of Pathology, Kidney Research Institute, \\ Medical Research Center, Seoul National University \\ College of Medicine, 103 Daehak-ro, Jongno-gu \\ Seoul 03080, Korea \\ Tel: +82-2-740-8380 \\ Fax: +82-2-743-5530 \\ E-mail: blue7270@snu.ac.kr
}

\begin{abstract}
Mucinous cystadenoma of the testis is a very rare tumor. Herein, we report a case of mucinous cystadenoma arising in the testis of a 61-year-old man, along with a literature review. Computed tomography showed a $2.5-\mathrm{cm}$-sized poorly enhancing cystic mass. Grossly, the tumor was a unilocular cystic mass filled with mucinous material and confined to the testicular parenchyma. Histologically, the cyst had a fibrotic wall lined by mucinous columnar epithelium without atypia. Immunohistochemical staining was positive for cytokeratin 20 and CDX2, as well as focally positive for cytokeratin 7 . The pathologic diagnosis was mucinous cystadenoma.
\end{abstract}

Key Words: Testis; Cystadenoma, mucinous; Immunohistochemistry
Testicular/paratesticular tumors resembling mucinous or serous ovarian tumors are rare; between the two subtypes, mucinous neoplasms are less commonly reported than serous neoplasms. ${ }^{1-7}$ To our knowledge, there have been approximately 24 case reports of mucinous neoplasms of the testis or paratestis, and only four cases of "testicular mucinous cystadenoma" were found in the English-language literature (Table 1). ${ }^{1,2,8-11}$ In Korea, two cases of mucinous cystadenomas of the paratestis or spermatic cord have been reported. ${ }^{4,7}$ However, there has been no published report of testicular mucinous cystadenoma in Korea. Here, we report a case of mucinous cystadenoma in the parenchyma of a testis.

\section{CASE REPORT}

A 61-year-old Korean man presented with a painless right testicular mass. Serum tumor markers including $\beta$-human chorionic gonadotrophin, $\alpha$-fetoprotein, and lactate dehydrogenase were within normal limits. Urine cytology was negative for malignant cells. Computed tomography (Fig. 1A) and ultrasound (Fig. 1B) imaging showed a 2.5-cm-sized poorly enhancing cystic mass at the right testis. Radical orchiectomy was performed.

Pathological examination demonstrated that the cystic mass was confined within the testicular parenchyma, with a size of $2.5 \times 2.4 \times 1.7 \mathrm{~cm}$. On macroscopic examination, the mass had a unilocular cavity filled with mucinous materials (Fig. 1C). The cyst wall was composed of thick fibrous tissue. There was no solid or papillary growth or calcification in the cyst.

Microscopically, the cyst was surrounded by a thick fibrous wall and was filled with mucinous material (Fig. 2A-C). The cyst had a single layer of columnar mucinous epithelial cells without nuclear atypia (Fig. 2D). Stromal mucin spillage was found around the tumor with inflammatory cell infiltration (Fig. 2E). The inflammatory cells were composed of lymphocytes, plasma cells, and histiocytes (Fig. 2F). There was no hemorrhage, necrosis, or calcification. Teratomatous elements, such as cartilage, bone, or other mesenchymal teratomatous compo- 
Table 1. Cases of primary testicular mucinous cystadenoma

\begin{tabular}{|c|c|c|c|c|c|}
\hline Age (yr) & Side & Maximum diameter $(\mathrm{cm})$ & Immunohistochemistry & Follow-up & Reference \\
\hline 61 & Rt & 2.5 & $\begin{array}{l}\text { CK7 (f+), CK20 (+), CDX2 (+), PAX8 (-), } \\
\text { Calretinin (-), D2-40 (-), WT-1 (-) }\end{array}$ & NDR 13 days & Current case \\
\hline 43 & Rt & 4.6 & ND & NDR $2.5 \mathrm{yr}$ & Shimbo et al. ${ }^{2}$ \\
\hline 35 & Rt & 9.0 & $\operatorname{AFP}(-)$, CA 19-9 (-), MUC2 (+), MUC5AC (-) & NDR 8 mo & Nokubi et al. ${ }^{9}$ \\
\hline 55 & Rt & 4.0 & CK7 (+), CK20 (+), MUC2 (+), MUC5AC (+) & NDR 5 mo & Naito et al. ${ }^{10}$ \\
\hline 39 & Rt & 2.0 & $\begin{array}{l}\text { CK7 (-), CK20 (+), CA125 (-), Chromogranin }(f+), \\
\text { Synaptophysin }(-)\end{array}$ & NDR 1 yr & Alasio et al..$^{11}$ \\
\hline
\end{tabular}

Rt, right; CK7, cytokeratin 7; f, focal; +, positive; CK20, cytokeratin 20; -, negative; NDR, no disease recurrence; ND, not none; AFP, $\alpha$-fetoprotein; CA 19-9, carbohydrate antigen 19-9; CA125, carbohydrate antigen 125.
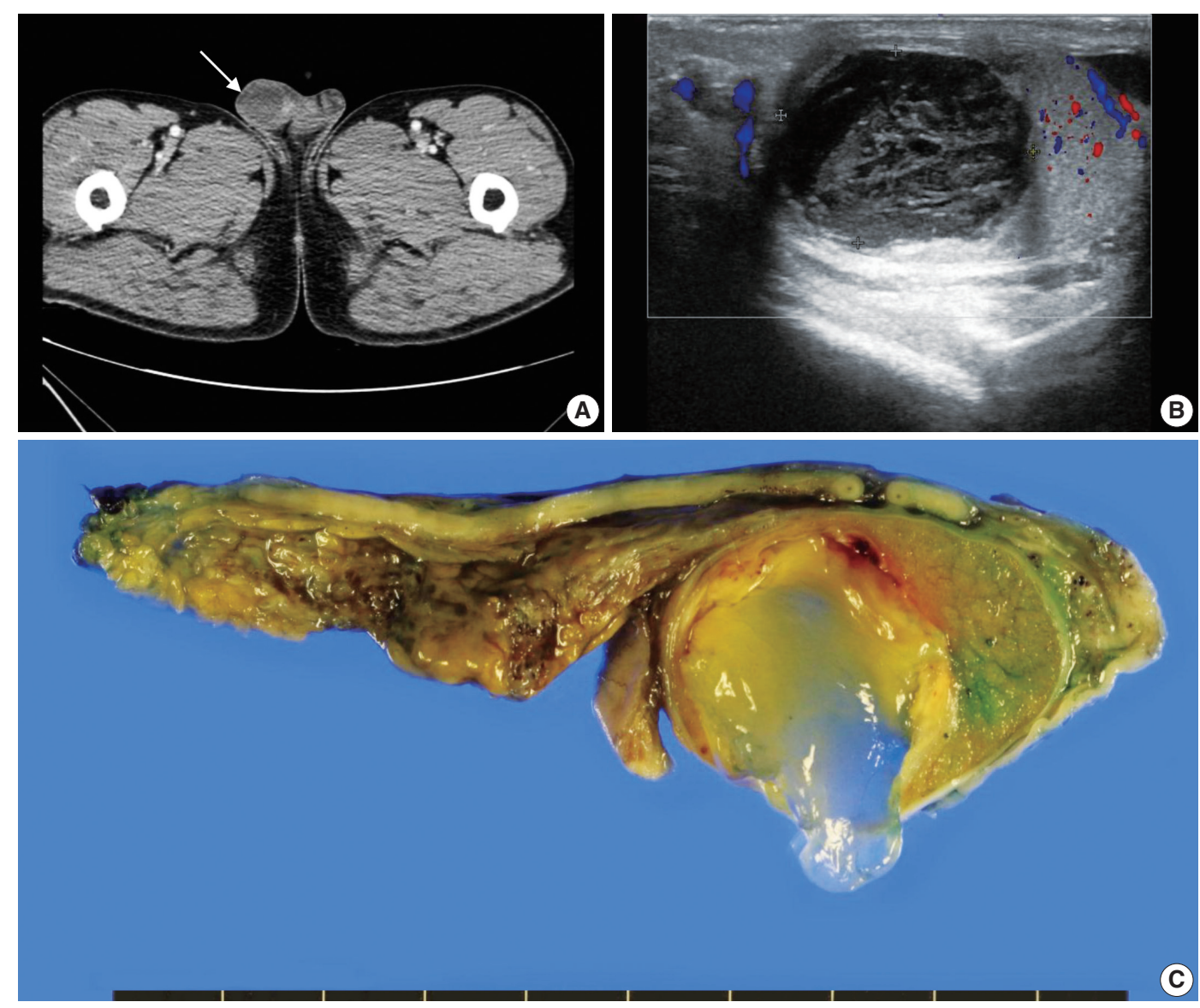

Fig. 1. Representative images of radiologic and macroscopic findings of a right testicular mass. Computed tomography (A) and ultrasound imaging (B) present a cystic mass (arrow in A) suspected to have a tumorous condition. (C) Surgical specimen reveals a mass with a unilocular cyst filled with mucinous materials.

nents, were not found. Squamous epithelium and intratubular germ cell neoplasia were also absent. Mitotic figures were not observed. Microscopically, the tumor was limited to the testicular parenchyma without involvement of the tunica albuginea.

Immunohistochemically, the lining epithelial cells were positive for cytokeratin 20 (CK20) (Fig. 3A) and CDX2 (Fig. 3C), as well as focally positive for cytokeratin 7 (CK7) (Fig. 3B). The cells were negative for PAX-8 (Fig. 3D), D2-40, WT-1, and cytokeratin 5/6 (CK5/6). Calretinin was weakly positive in cytoplasm.

This study was approved by the Institutional Review Board (IRB) of Seoul National University Hospital.

\section{DISCUSSION}

Mucinous neoplasms of the testis or paratestis are rare and 

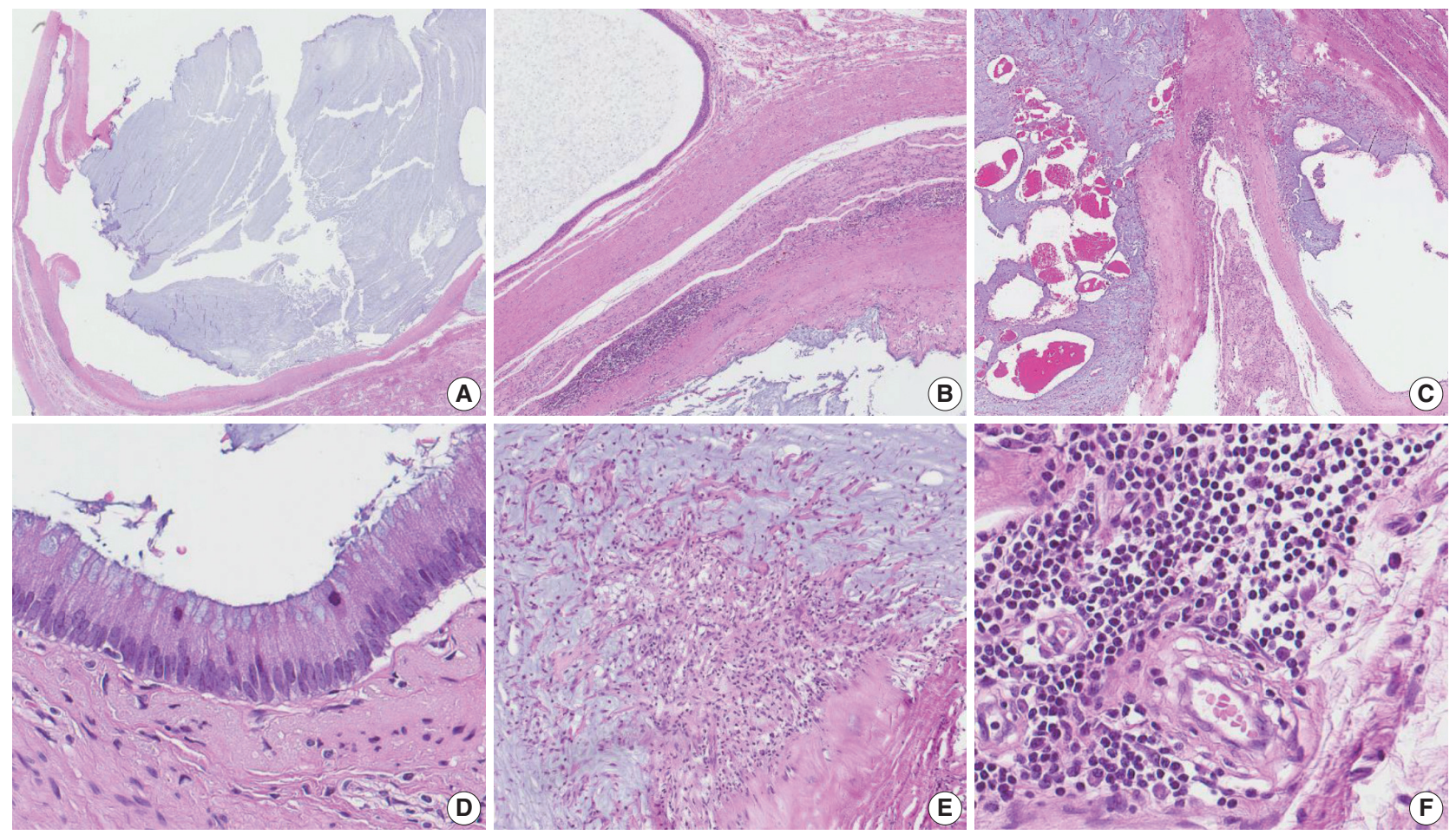

Fig. 2. Microscopic findings of the mucinous cystadenoma of the testis. (A) The tumor has a uniloculated cyst with an expanding growth pattern. (B) The cyst is surrounded by a thick fibrous capsule, and there are some inflammatory cells around the tumor. (C) The cyst is filled with mucinous material. (D) The wall of the cavity is lined by a single layer of columnar mucinous epithelium, and the lining cells show no cytologic atypia. (E) Mucin extravasation into the stroma and some inflammatory cell infiltration are observed. (F) The inflammatory cells are composed of lymphocytes, plasma cells, and histiocytes.

not well described in the medical literature. ${ }^{8,12}$ The first reported case of mucinous neoplasm was mucinous cystadenoma in the paratestis of an 11-year-old boy by Kellert in 1959. ${ }^{1}$ To date, most reported testicular/paratesticular ovarian-type surface epithelial neoplasms have been of the "serous" subtype, ${ }^{5,13,14}$ and "intratesticular" neoplasms have rarely been reported. ${ }^{12}$ Previous papers reported approximately 24 cases of mucinous neoplasms of the testis or paratestis; among these, only four reports described "testicular mucinous cystadenoma" excluding the present case. $^{1,2,8,9}$ In the Korean literature, three cases of serous borderline tumors of the testis/paratestis and two cases of paratesticular mucinous cystadenomas were found, but there has been no report of any "mucinous cystadenoma of the testicular parenchyma" to date. ${ }^{3-7}$

The histologic features of mucinous cystadenomas of the testis resemble those of common ovarian mucinous cystadenomas. ${ }^{1,2}$ That is, the cyst is typically composed of mucinous epithelium with tall, columnar endocervical-like cells lacking nuclear atypia. ${ }^{15}$ However, there are several histologic differences between testicular mucinous cystadenomas and ovarian mucinous cystadenomas. Testicular mucinous cystadenomas are generally not as large as ovarian tumors, purportedly because the testicular tumors are found by patients earlier than their ovarian counterparts due to the sites at which the tumors arise. Testicular tumors are more often unilocular and more frequently exhibit mucus extravasation associated with fibrosis or calcification than ovarian tumors, possibly because of trauma due to their superficial location. ${ }^{15}$

The origin of testicular mucinous neoplasms has not yet been clarified; thus, several hypotheses have been suggested. Ulbright and Young ${ }^{15}$ supported the theory that the tumors arise from metaplasia of the mesothelium of the visceral tunica vaginalis. Another theory, suggested by Shimbo et al., ${ }^{2}$ is that inflammation results in mesothelial introduction into the testicle and mucinous metaplasia. The theory that the tumors arise from the remnants of the Müllerian ducts persisting in the male appendix, testis, or extratesticular scrotal contents is considered the most reasonable by some researchers. ${ }^{8,16}$ Others have postulated the possibility of one-sided teratoma cell differentiation., ${ }^{9,15,17}$

Differential diagnoses include testicular mucinous borderline neoplasms/carcinomas, germ cell tumors, mesotheliomas, and metastatic mucinous tumors. ${ }^{15}$ In comparison with mucinous borderline tumors or mucinous carcinomas, mucinous cystade- 

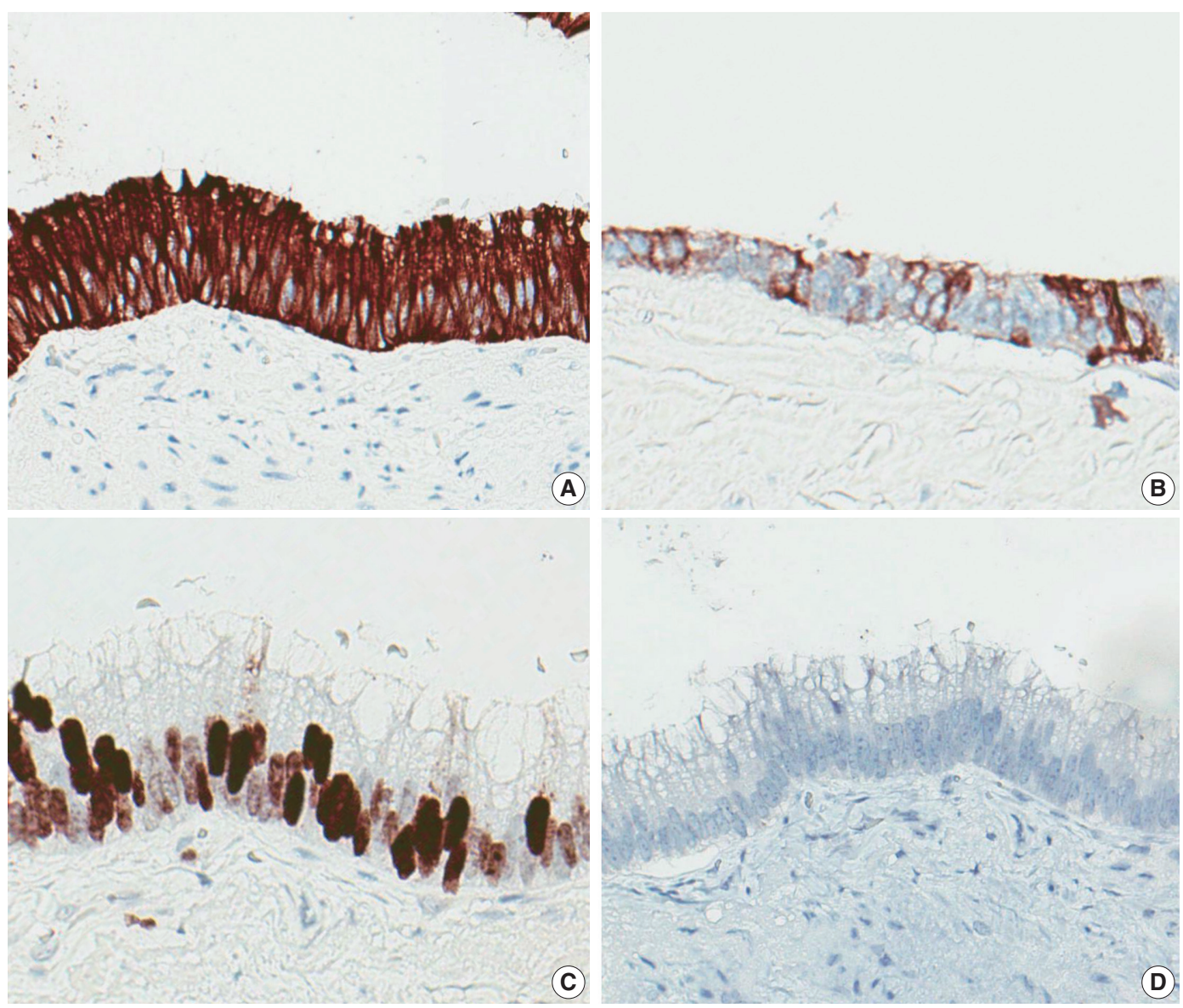

Fig. 3. Immunohistochemical staining results. The lining cells are positive for cytokeratin 20 (A), focally positive for cytokeratin 7 (B), positive for CDX2 (C), and negative for PAX8 (D).

nomas lack the atypia of tumor cells. ${ }^{8,15}$ Testicular mucinous carcinomas or borderline neoplasms have been reported more frequently than testicular mucinous cystadenomas. To date, seven mucinous borderline neoplasms and four mucinous carcinomas have been reported. ${ }^{1,8}$ It is also important to rule out germ cell tumors, for example, teratomas with prominent mucinous components. Teratomas are known to occur in patients with a median age of 23-29 years. Teratomas almost always have components other than mucinous epithelial lined cysts, and $90 \%$ of those tumors occurring in adults are accompanied by intratubular germ cell neoplasia of unclassified type..$^{15}$ Unlike testicular mucinous cystadenomas, mesotheliomas exhibit positivity for calretinin, which is known to be expressed in mesothelial cells. ${ }^{10}$ Pathologists must consider metastatic mucinous tumors. The appropriate immunohistochemical staining, cytologic atypia, and history of mucinous carcinoma are critical for determining the origin of the tumor.

The immunohistochemical profile of the tumor has not been specified until now. ${ }^{8}$ However, in several reports, testicular muci- nous neoplasms have shown immunostaining that is positive for both CK7 and CK20 or positive for CK20 and negative for $\mathrm{CK} 7{ }^{8}$ The present case showed positive staining for $\mathrm{CK} 20$ and focal positive staining for $\mathrm{CK} 7$, a result similar to previous studies. Additionally, the present case also showed positive staining for CDX2 and negative staining for PAX8, D2-40, and CK5/6. Calretinin immunostaining was weak only in the cytoplasm. CDX2 is known to be useful in distinguishing primary ovarian mucinous tumors from metastases of lower gastrointestinal tract origin. ${ }^{18}$ There has been only one case report of CDX2 expression in testicular or paratesticular mucinous neoplasms, revealing CDX2-positive and PAX8-negative staining. ${ }^{4}$ Together with the results of this report, testicular or paratesticular mucinous tumors may express CDX2. Immunohistochemical results for calretinin, CK5/6, D2-40, and PAX8 suggest that testicular mucinous cystadenoma does not originate from mesothelium or Müllerian duct remnants, while the results support the possibility of monodermal teratoma differentiation.

Although only a small number of testicular mucinous cyst- 
adenomas have been reported, these reported cases have shown good prognosis, similar to the prognosis of ovarian mucinous cystadenomas. ${ }^{1}$

In conclusion, mucinous cystadenoma of the testis is an extremely rare benign tumor and likely expresses CDX2.

\section{Conflicts of Interest}

No potential conflict of interest relevant to this article was reported.

\section{REFERENCES}

1. Elliott JE, Klein JR, Drachenberg DE. Primary testicular mucinous neoplasms: case report and literature review. Can Urol Assoc J 2010; 4: E112-5.

2. Shimbo M, Araki K, Kaibuchi T, Kuramochi H, Mori I. Mucinous cystadenoma of the testis. J Urol 2004; 172: 146-7.

3. Park KM, Cho NB, Song KY. A serous papillary cystadenoma of low malignant potential in paratesticular tissue. Korean J Pathol 1996; 30: 463-5.

4. Kim JY, Lee YT, Kang HJ, Lee CH. Primary mucinous cystadenoma of the spermatic cord within the inguinal canal. Diagn Pathol 2012; 7: 139 .

5. Park MI, Kim HS, Suh KS, Kang DY. Paratesticular papillary serous tumor of low malignant potential: a case report. Korean J Pathol 2004; 38: 427-9.

6. Park HJ, Park SW, Chon WH, Jung SG, Park NC. A serous papillary cystadenoma of borderline malignancy in testis. Korean J Androl 2007; 25: 36-8.

7. Weon HC, Kim IS, Jang CS, Hong SJ, Lee MS. Papillary mucinous cystadenoma, paratesticular: a case report. Korean J Urol 1986; 27: 359-60.

8. Celdran JO, Rodriguez CS, Valverde FM, Compiano LO. Primary mucinous cystadenocarcinoma of the testis: an extremely rare ovarian-type surface epithelial carcinoma. J Cancer Res Ther 2015;
11: 647 .

9. Nokubi M, Kawai T, Mitsu S, Ishikawa S, Morinaga S. Mucinous cystadenoma of the testis. Pathol Int 2002; 52: 648-52.

10. Naito S, Yamazumi K, Yakata Y, et al. Immunohistochemical examination of mucinous cystadenoma of the testis. Pathol Int 2004; 54: 355-9.

11. Alasio TM, Borin J, Taylor K, Bar-Chama N, Unger PD. Intratesticular mucinous cystadenoma: immunohistochemical comparison with ovarian and colonic tissue. Arch Pathol Lab Med 2005; 129: 399-402.

12. Burger T, Schildhaus HU, Inniger R, et al. Ovarian-type epithelial tumours of the testis: immunohistochemical and molecular analysis of two serous borderline tumours of the testis. Diagn Pathol 2015; 10: 118.

13. Olla L, Di Naro N, Puliga G, Tolu GA. Intraparenchymal serous papillary cystadenoma of the testis: a case report. Pathologica 2013; 105: 15-7.

14. Remmele W, Kaiserling E, Zerban U, et al. Serous papillary cystic tumor of borderline malignancy with focal carcinoma arising in testis: case report with immunohistochemical and ultrastructural observations. Hum Pathol 1992; 23: 75-9.

15. Ulbright TM, Young RH. Primary mucinous tumors of the testis and paratestis: a report of nine cases. Am J Surg Pathol 2003; 27: 1221-8.

16. Amin MB. Selected other problematic testicular and paratesticular lesions: rete testis neoplasms and pseudotumors, mesothelial lesions and secondary tumors. Mod Pathol 2005; 18 Suppl 2: S131-45.

17. Young RH, Scully RE. Testicular and paratesticular tumors and tumor-like lesions of ovarian common epithelial and mullerian types: a report of four cases and review of the literature. Am J Clin Pathol 1986; 86: 146-52.

18. Vang R, Gown AM, Wu LS, et al. Immunohistochemical expression of CDX2 in primary ovarian mucinous tumors and metastatic mucinous carcinomas involving the ovary: comparison with CK20 and correlation with coordinate expression of CK7. Mod Pathol 2006; 19: $1421-8$ 